\title{
Equilibria in structured populations
}

\author{
J. M. Cushing*
}

Department of Mathematics, Building 89, University of Arizona, Tucson, Arizona 85721, USA

\begin{abstract}
The existence of a stable positive equilibrium state for the density $\rho$ of a population which is internally structured by means of a single scalar such as age, size, etc. is studied as a bifurcation problem. Using an inherent birth modulus $n$ as a bifurcation parameter it is shown for very general nonlinear model equations, in which vital birth and growth processes depend on population density, that a global unbounded continuum of nontrivial equilibrium pairs $(n, \rho)$ bifurcates from the unique (normalized) critical point $(1,0)$. The pairs are locally positive and conditions are given under which the continuum is globally positive. Local stability is shown to depend on the direction of bifurcation. For the important case when density dependence is a nonlinear expression involving a linear functional of density (such as total population size) it is shown how a detailed global bifurcation diagram is easily constructed in applications from the graph of a certain real valued function obtained from an invariant on the continuum. Uniqueness and nonuniqueness of positive equilibrium states are studied. The results are illustrated by several applications to models appearing in the literature.
\end{abstract}

Keywords: Structured populations - Equilibria - Stability - Bifurcation

\section{Introduction}

Consider a population structured in the sense that its density is a function $\rho=\rho(t, a) \geqslant 0$ of time $t$ and a real variable $a$ which categorizes individuals according to some physical characteristic such as chronological age, physical size, mass, volume, concentration of some metabolically important chemical, etc. The number of individuals of types between $a_{1}$ and $a_{2}$ at time $t$ is then $\int_{a_{1}}^{a_{2}} \rho(t, a) d a$. The dynamics of $\rho$ are described by the familiar equation

$$
\partial_{t} \rho+\partial_{a}(v \rho)+\delta=0, \quad t>t_{0}, \quad a>a_{0}
$$

where $d a / d t=v$ describes the dynamics of the characteristic $a$ and where $\delta$ is an $a$-specific growth law (e.g. see $[28,36,41,44]$ ). Here $t_{0}$ is some initial time and $a_{0}$ is the characteristic for neonates which by rescaling we assume without

\footnotetext{
* This research was done while the author was on leave at the Lehrstuhl für Biomathematik, Universität Tübingen, Auf der Morgenstelle 10, 7400 Tübingen 1, Federal Republic of Germany
} 
loss in generality are $t_{0}=0, a_{0}=0$. In general $\delta$ depends on $t, a$ and in density dependent dynamics also on $\rho$. Equations of this type have been used in a variety of disciplines including population dynamics $[20,46]$, cell growth dynamics $[27,36,37,44]$, epidemiology $[13,21]$ amongst others.

Generally there are several side conditions associated with (1.1): a "birth" or "recruitment" rate $\left.\nu \rho\right|_{a=0}=\beta$ in which $\beta$ can depend on $t$, $a$ and $\rho$; the requirement that no individual has a characteristic type greater than some maximum $\rho(t, a)=0, t>0, a \geqslant A>0$; and the prescription of an initial density $\rho(0, a)$. The well-posedness of this problem has been considered in various contexts by several authors (e.g. see $[9,16,26,46]$ ).

Of special interest in applications are the asymptotic states (as $t \rightarrow+\infty$ ) of solutions of (1.1). In particular if the equations are autonomous, i.e. if $\delta, \beta$ and $v$ do not depend explicitly on time $t$, equilibrium solutions $\rho=\rho(a) \geqslant 0$ are of fundamental importance and it is with the existence and stability of such solutions with which this paper is concerned. Specifically we will study the existence and stability of solutions $\rho=\rho(a) \geqslant 0$ of the equations

$$
\begin{gathered}
\partial_{t} \rho+\partial_{a}(v \rho)+\delta=0, \quad t>0, \quad 0<a<A \\
\left.\nu \rho\right|_{a=0}=\beta \text { and } \rho(t, a)=0 \quad \text { for } t>0, \quad a \geqslant A
\end{gathered}
$$

where $\delta$ and $\beta$ will be allowed to depend in a very general way on $a$ and $\rho$, except that it is required that both identically vanish when $\rho \equiv 0$. This means growth and birth processes cease when density drops to zero and in particular there is no seeding/harvesting or immigration/emigration (which is independent of population density). Under this assumption $\rho \equiv 0$ is a trivial equilibrium state.

In this paper the existence of equilibria will be studied as a bifurcation phenomenon, namely equilibrium solutions will be sought which bifurcate from $\rho \equiv 0$ at a critical value of a selected bifurcation parameter. In theoretical population dynamics mathematical models are often studied with reference to a parameter identified as a "birth", "fertility" or "maternity" modulus or a "net reproductive" rate. In keeping with this point of view we normalize in a certain way (see (3.1) below) the linear birth law $\beta^{\prime}(\rho)$ obtained from the linearization of $\beta$ at $\rho \equiv 0$ and write $\beta^{\prime}(\rho)=n m(\rho)$ where $n$ is a real parameter which we call the "inherent birth modulus". Thus we will write $\beta=n m+h_{1}$ in (1.2) where $h_{1}$ is of order greater than one in $\rho$ near 0 .

With regard to the growth rate $\delta$ we are motivated by the frequently considered form $\delta=\rho D$ where $D$ is a per unit density growth rate which in general depends on $a$ and $\rho$. If $D$ is written, without loss in generality, as $D=\mu+d(\rho)$ where $d(0)=0$ and $\mu$ is independent of $\rho$ then $\delta=\rho \mu+h_{2}$ where $h_{2}=\rho d(\rho)$ is of order greater than one in $\rho$ near 0 . For added generality we allow for a more general form for $h_{2}$ so long as it is of order higher than one at $\rho \equiv 0$.

In summary we will be concerned with positive solutions $\rho=\rho(a)>0,0 \leqslant a<$ $A$, of the equilibrium equations

$$
\begin{aligned}
& \text { (a) } \partial_{a}(v \rho)+\mu \rho+h_{2}(n, \rho)=0, \quad 0<a<A \\
& \text { (b) } \nu(0) \rho(0)=n m(\rho)+h_{1}(n, \rho) \\
& \text { (c) } \rho(a)=0, \quad a \geqslant A
\end{aligned}
$$


where $n \in R$ is a real, $m$ is a linear functional and the $h_{i}$ are of order greater than one at $\rho \equiv 0$. The question is: for what values of the inherent birth modulus $n$ do there exist stable positive solutions of (1.3)?

By the change of variables $a \rightarrow \int_{0}^{a}(1 / v(s)) d s, \rho \rightarrow v \rho$ one could achieve $v \equiv 1$. I prefer however to retain the general form (1.3) and the explicit appearance of $v$ in the results below.

A frequently occurring case is when $\delta, \beta$ have the form

$$
\delta=\rho D(\rho), \quad \beta=\int_{0}^{A} \rho F(\rho) d a
$$

where $D, F$ are per unit growth and fertility coefficients respectively. We will pay close attention to this important special case.

There are many papers which deal with the existence and stability of equilibria for the case (1.4) with $v \equiv 1$ using a variety of techniques and requiring various restrictions on $D$ or $F$ such as boundedness, monotonicity, Lipschitz or differentiability conditions or special types of dependencies on $\rho$ (e.g. see $[10,11,14$, $16,24,25,30-32,34,35,39,40,47])$. By contrast our most general results require only continuity assumptions in (1.3). Moreover they considerably generalize and extend the results for this special case obtained in $[5,6]$ using bifurcation techniques.

After a development of the necessary linear theory in Sect. 2 it will be shown in Sect. 3 that the equilibrium equations (1.3) under only continuity conditions possess an unbounded continuum of nontrivial equilibrium pairs $(n, \rho)$ which bifurcates from a unique trivial pair $\left(n_{0}, 0\right)$ (when $m$ is normalized then $n_{0}=1$ ) which at least near this bifurcation point consists of positive pairs, i.e. $\rho(a)>0$ for $0 \leqslant a<A$. In Sect. 4 it will be seen that in general this continuum may not globally consist of positive equilibrium pairs, although for the important special case when $\delta=\rho D$ this is shown to be true. Furthermore the trivial equilibrium $\rho \equiv 0$ will be shown in Sect. 5 to lose stability as $n$ increases through $n_{0}=1$ while the bifurcating positive equilibria are stable in the event of supercritical bifurcation and unstable in the event of subcritical bifurcation. In general stability, when it occurs, may not persist globally along the continuum however [4].

Some results concerning the "spectrum" of $n$ values associated with the continuum of positive equilibrium pairs are derived in Sect. 6 using a certain invariant. In Sect. 7 the important special case when $\delta$ and $\beta$ depend upon an arbitrary linear functional of density is studied in more detail and results concerning the spectrum, uniqueness and nonuniqueness are obtained. It is shown how the simple graphing of a certain real valued function yields a bifurcation diagram from which specific information concerning the spectrum and the boundedness and uniqueness of positive equilibria can be obtained. Some illustrative examples and applications are given in Sect. 8 in which detailed bifurcation results are obtained for many types of models found in the literature.

\section{Linear equations}

Let $C_{0}(A)$ be the Banach space of continuous functions $v:[0, A] \rightarrow R$ under the usual sup-norm $|v|_{0}$ and let $L(A)$ be the space of Lebesgue integrable functions on $[0, A]$. Let $\Delta(A)$ denote the set of pairs $(\mu, v)$ where $v \in C_{0}(A)$ is positive and 
$\mu:[0, A) \rightarrow R$ is continuous and where $v(0)=1$,

$$
M(A-)=+\infty \quad \text { where } M(a):=\int_{0}^{a}(\mu(s) / v(s)) d s .
$$

Let $V(a):=\int_{0}^{a}(1 / v(s)) d s$ and $v_{m}:=\min v>0$. Define the continuous function

$$
\rho_{0}(a):= \begin{cases}\frac{1}{v(a)} \exp (-M(a)), & 0 \leqslant a<A \\ 0, & A \leqslant a .\end{cases}
$$

The linear space $B=B(\mu, v)$ of functions $\rho=\rho(a)$, continuous on $R^{+}=[0,+\infty)$ and satisfying $\rho(a)=0$ for $a \geqslant A$, for which $\rho(a) / \rho_{0}(a)$ is continuous on $[0, A]$ is a Banach space under the norm $|\rho|_{\mu}:=\left|\rho / \rho_{0}\right|_{0}$. Let $P=P(\mu, v):=R \times B(\mu, v)$ have the norm $|(n, \rho)|=|n|+|\rho|_{\mu}$. In later sections the sets $B^{+}=B^{+}(\mu, v):=$ $\{\rho \in B \mid \rho>0$ on $[0, A)\}$ and $\Delta^{+}(A):=\{(\mu, v) \in \Delta(A) \mid \mu \geqslant 0$ on $[0, A)\}$ will be needed. Note that $\rho_{0} \in B^{+}$. Consider the linear nonhomogeneous equations

$$
\begin{aligned}
& \text { (a) } \partial_{a}(v \rho)+\mu \rho=g_{2}, \quad 0<a<A \\
& \text { (b) } \rho(0)=n m(\rho)+g_{1}
\end{aligned}
$$

for $n \in R$ and $g:=\left(g_{1}, g_{2}\right) \in P$ under the hypothesis

$$
(\mu, v) \in \Delta(A) \quad \text { and } \quad m: B(\mu, v) \rightarrow R \text { is linear and bounded. }
$$

By a solution of (2.2) in $B$ is meant a function $\rho \in B$ for which $v \rho$ is differentiable for $0<a<A$ and the Eqs. (2.2) hold. The homogeneous version of (2.2)

$$
\begin{aligned}
& \text { (a) } \partial_{a}(v \rho)+\mu \rho=0, \quad 0<a<A \\
& \text { (b) } \rho(0)=n m(\rho)
\end{aligned}
$$

has the solution $\rho=\rho_{0}$ in $B$ as given by (2.1) if $n m\left(\rho_{0}\right)=1$.

The general solution in $B$ of Eq. $(2.2 \mathrm{a})$ is

$$
\rho(a)= \begin{cases}\rho_{0}(a)\left[k+\int_{0}^{a}\left(g_{2}(s) / \rho_{0}(s) v(s)\right) d s\right], & 0 \leqslant a<A \\ 0, & A \leqslant a\end{cases}
$$

for arbitrary $k \in R$. Equation (2.2b) is satisfied if and only if $k$ satisfies the equation

$$
\left(1-n m\left(\rho_{0}\right)\right) k=\Omega(g):=g_{1}+n m\left(\rho_{0}(a) \int_{0}^{a}\left(g_{2} / \rho_{0} v\right) d s\right) .
$$

The linear functional $\Omega: P \rightarrow R$ is easily seen to be bounded under (2.3) for each fixed $n \in R$.

If $n m\left(\rho_{0}\right) \neq 1$ then $k=\Omega(g) /\left(1-n m\left(\rho_{0}\right)\right)$ substituted into the RHS of (2.5) yields a solution operator $S: P \rightarrow B$ where $\rho=S(g)$ is the unique solution of (2.2) in $B$. It is easy to see that $S$ is linear and bounded.

If $n m\left(\rho_{0}\right)=1$ then (2.2) is solvable for $k$ if and only if $\Omega(g)=0$, i.e. $g \in P_{0}$ where $P_{0}=P_{0}(\mu, v)$ is the Banach subspace of $g \in P$ satisfying $\Omega(g)=0$. When $g \in P_{0}$ then (2.2) has a unique solution in $B_{0}=B_{0}(\mu, v)$, the Banach subspace of 
those $\rho \in B$ satisfying $\int_{0}^{A} \rho \rho_{0} d a=0$, which is given by (2.5) with

$$
k=-\int_{0}^{A} \rho_{0}^{2}(a) \int_{0}^{a} g_{2}(s) / \rho_{0}(s) v(s) d s d a / \int_{0}^{A} \rho_{0}^{2}(a) d a .
$$

This $k$, when substituted into the RHS of (2.5), yields a solution operator $S_{0}: P_{0} \rightarrow B_{0}$ where $\rho=S_{0} g$ is the unique solution of $(2.2)$ in $B_{0}$. The operator $S_{0}$ is linear and bounded.

Lemma. Assume (2.3). If the homogeneous system (2.4) has no nontrivial solutions in $B(\mu, v)$ (i.e. if $n m\left(\rho_{0}\right) \neq 1$ ) then the nonhomogeneous system (2.2) has a unique solution $\rho=S g$ in $B$ for every $g \in P(\mu, v)$ where the operator $S: P \rightarrow B$ is linear and compact. On the other hand if (2.4) has nontivial solutions in $B$ (i.e. if $n m\left(\rho_{0}\right)=1$ ) then (2.2) has a solution in $B$ if and only if $g \in P_{0}(\mu, v)$ in which case there is $a$ unique solution $\rho=S_{0} g$ in $B_{0}(\mu, v)$ where $S_{0}: P_{0} \rightarrow S_{0}$ is linear and compact.

Proof. All that remains to be shown is that the bounded linear operators $S, S_{0}$ are compact. Let $g^{j} \in P$ be a bounded sequence $\left|g^{j}\right| \leqslant 1$ and denote $\rho^{j}=S g^{j}$. Then the sequence $\rho^{j}$ is bounded in $B$. We wish to show that there is a subsequence convergent in $B$. Since $S$ is bounded it follows that the sequence of functions $z^{j}:=\rho^{j} / \rho_{0}$, continuous on $[0, A]$, is uniformly bounded with respect to the sup-norm $|\cdot|_{0}$. Furthermore, a calculation shows that $\partial_{a} z^{j}=g_{2}^{j} / \rho_{0} v$ and since $g_{2}^{j} \in B$ it follows that $z^{j}$ is continuously differentiable. The sequence of derivatives is uniformly bounded since $\left|\partial_{a} z^{j}\right|_{0} \leqslant\left|g_{2}^{j}\right|_{\mu} / v_{m} \leqslant\left|g^{j}\right| / v_{m} \leqslant 1 / v_{m}$ and hence the Ascoli-Arzela theorem yields a subsequence which converges with respect to the sup-norm to a limit function $\hat{z}$ which is continuous on $[0, A]$. Define $\hat{\rho}:=\hat{z} \rho_{0}$ and relabel, if necessary, the convergent subsequence as $z^{j}$. Then $\left|\rho^{j}-\hat{\rho}\right|_{\mu}=\left|z^{j}-\hat{z}\right|_{0} \rightarrow 0$.

A similar proof works for $S_{0}$.

\section{Bifurcating branches of nontrivial equilibria}

By an equilibrium pair $(n, \rho)$ of $(1.2)$ is meant a pair $(n, \rho) \in P$ for which $v \rho$ is differentiable on $(0, A)$ and the equilibrium equations (1.3) are satisfied with the value $n$. A positive equilibrium pair is an equilibrium pair in $P^{+}=P^{+}(\mu, v):=$ $R \times B^{+}(\mu, v)$ and a nontrivial pair is one for which $\rho \neq 0$.

In addition to (2.3) it will be assumed that $m\left(\rho_{0}\right) \neq 0$. The linear term $m$ can be referred to as the inherent (normalized) birth law in that it describes births in the absence of density effects. This assumption means that this inherent birth law is not zero, at least at density $\rho_{0}$, i.e. that births do occur when density effects on birth processes are absent. In most applications $m$ is a positive functional, i.e. $m(\rho)>0$ for $\rho \geqslant 0(\not \neq 0)$, in which case this condition holds. By rescaling $n$ and $a$ if necessary we can assume without loss in generality that

$$
m\left(\rho_{0}\right)=1 \text { and } v(0)=1 .
$$

With regard to the higher order terms $h_{i}$ in the equilibrium equations (1.3) the following hypotheses will be assumed throughout.

H1. $\delta=\mu \rho+h_{2}(n, \rho), \beta=n m(\rho)+h_{1}(n, \rho)$ where $v, \mu$ and $m$ satisfy (2.3) and (3.1) and where $h_{1}: P(\mu, v) \rightarrow R, h_{2}: P(\mu, v) \rightarrow B(\mu, v)$ are continuous, take 
bounded sets to bounded sets and are of order $o\left(|\rho|_{\mu}\right)$ near $\rho=0$ uniformly on bounded $n \in R$ sets.

Under $H 1,(n, 0)$ is an equilibrium pair for all $n \in R$. We will also need

$H 2$. In hypothesis $H 1$ the $h_{i}$ are $q \geqslant 2$ times continuously Fréchet differentiable near $(n, \rho)=(1,0)$.

It is well known in bifurcation theory that the only possible bifurcation points $(n, 0)$ of the equilibrium equations (1.3) are those for which $n$ yields a linearized problem (2.4) which has a nontrivial solution. The linear theory of Sect. 2 shows that the only possible such bifurcation point is $(1,0)$. The following theorem asserts that bifurcation indeed occurs at $(1,0)$.

Let $E$ denote the set of nontrivial equilibrium pairs of (1.3) and let $c l(E)$ denote its closure in $P$. A subcontinuum of $c l(E)$ is a subset which is closed and connected in $P$.

Theorem 1 (Existence). (i) Assume H1. Then $c l(E)=E \cup\{(1,0)\}$ contains two unbounded subcontinua $C^{+}$and $C^{-},(1,0) \in C^{ \pm}$, which in a sufficiently small neighbourhood of $(1,0)$ consist, for $\varepsilon>0$ and $\varepsilon<0$ respectively, of equilibrium pairs of the form

$$
\rho=\rho(\varepsilon)=\varepsilon \rho_{0}+\varepsilon w(\varepsilon), \quad n=n(\varepsilon)=1+\gamma(\varepsilon), \quad 0 \leqslant|\varepsilon|<\varepsilon_{0}
$$

for some $\varepsilon_{0}>0$ where $w \in B_{0},|w|_{\mu}=0(|\varepsilon|)$ and $|\gamma|=0(|\varepsilon|)$ near $\varepsilon=0$.

(ii) Assume H2. Then $w:\left(-\varepsilon_{0}, \varepsilon_{0}\right) \rightarrow B_{0}, y:\left(-\varepsilon_{0}, \varepsilon_{0}\right) \rightarrow R$ are $q \geqslant 1$ times continuously differentiable and

$$
\gamma_{1}:=\partial_{\varepsilon} \gamma(0)=\frac{1}{2} m\left(\rho_{0}(a) \int_{0}^{a} \partial_{\rho}^{2} h_{2}(1,0)\left(\rho_{0}, \rho_{0}\right) / \rho_{0} v d s\right)-\frac{1}{2} \partial_{\rho}^{2} h_{1}(1,0)\left(\rho_{0}, \rho_{0}\right) .
$$

In the formula $(3.3), \partial_{\rho}^{2} h_{i}(1,0)(\cdot, \cdot)$ denotes the second order Fréchet derivative of $h_{i}(n, \rho)$ with respect to $\rho$ at $(n, \rho)=(1,0)$. The proof of this theorem is given in the Appendix.

Remarks. (1) If the $h_{i}$ are not globally defined on $P$ but satisfy $H 1$ only on a bounded domain $D \subset P$ containing $(1,0)$ then both $C^{+}$and $C^{-}$in Theorem 1 , rather than being unbounded in $P$, instead meet the boundary of $D$ [33].

(2) Theorem 1 does not exclude the possible existence of other nontrivial equilibrium pairs which do not lie on a continuum bifurcating from $(1,0)$. For an example see [5].

(3) The number $\gamma_{1}$, when nonzero, determines the direction of bifurcation as $\varepsilon$ increases through 0 in that $\gamma_{1}>0$ (resp. $<0$ ) implies that $n=n(\varepsilon)$ is increasing (resp. decreasing) near $\varepsilon=0$.

(4) If $\delta, \beta$ have the form (1.4) and $D(\rho)=\mu \rho+d(\rho), f=\phi+r(\rho)$, where $\phi \in L(A), \int_{0}^{A} \phi \rho_{0} d a=1$ and where $d: B \rightarrow C_{9}(A), r: B \rightarrow L(A)$ are continuous, then $H 1$ holds with $h_{2}=\rho d(\rho), h_{1}=n \int_{0}^{A} \rho r(\rho) d a$. Theorem 1(ii) applies when $d$ and $r$ have two or more continuous derivatives near $\rho \equiv 0$. A careful reading of the proof shows however that in this case Theorem 1(ii) remains valid if $d$ and $r$ are only once continuously differentiable near $\rho \equiv 0$. 
Theorem 1 shows that under very general conditions the equations (1.2) for structured population growth always possess a "global" continuum of nontrivial equilibria which bifurcates from a unique value of the inherent birth modulus $n$. Next we consider the important problems of the positivity and stability of the equilibria from the continua in Theorem 1.

\section{Positive equilibrium pairs}

It is not difficult to see from the definition of the norm $|\cdot|_{\mu}$ that any $\rho \in B$ close to $\rho_{0}$ is positive on $[0, A)$. In fact $\left|\rho_{0}-\rho\right|_{\mu}=\left|1-\rho / \rho_{0}\right|_{0}<1$ implies $\rho(a)>0$ on $[0, A)$. Thus the nontrivial equilibrium pairs $(n, \rho) \in C^{+}$are positive for $\varepsilon>0$ small enough. (Similarly those on $C^{-}$are negative.) The continuum $C^{+}$exists globally and it is natural to ask whether all nontrivial equilibrium pairs from $C^{+}$ are positive. That the answer to this question is in general "no" can be seen from the following example.

Example. Take $v \equiv 1, A=1$ and $\mu=1 /(1-a)$. Let $m(\rho)=\rho(0), h_{1}=-n \kappa \rho^{2}(0)$, $0<\kappa \in R$, and $h_{2}=(1-a) I^{2}(1) I(a)$ where $I(a)=\int_{0}^{a} \rho(s) /(1-s) d s$. Then $\rho_{0}(a)=$ $1-a$ and $H 2$ holds (for any integer $q$ ). Theorem 1 implies that an unbounded continuum $C^{+}$of equilibrium pairs solving $(1.3)$ and containing $(1,0)$ exists which near $(1,0)$ consists of positive equilibria. This example can however be solved explicitly.

Substitute $I \in R$ for $I(1)$ and set $z=\rho /(1-a)$. A differentiation of (1.3a) by $a$ yields the equation $z^{\prime \prime}+I^{2} z=0$. Since $z(0)=\rho(0), \partial_{a} z(0)=0$ it follows that $z=\rho(0) \cos I a$ or $\rho=\rho(0)(1-a) \cos I a$. But $(1.3 \mathrm{~b})$ in this example implies $\rho(0)=$ $(n-1) / n \kappa$ so that $\rho(a)=(n-1)(1-a)(n \kappa)^{-1} \cos I a$. This is a solution of the equilibrium equations (1.3) if and only if $I(1)=I$, i.e. if and only if $0<I \in R$ solves the equation $(n-1) \sin I=n \kappa I^{2}$. A straightforward graphical investigation of this equation shows that there exists a smallest solution $I=I(n)>0$ for all $n>1$ with the following properties:

$$
I(n) \text { is strictly increasing, } I(1)=0, I(+\infty)=I_{0}<+\infty .
$$

Moreover $I_{0}>\pi / 2$ if and only if

$$
\kappa<(2 / \pi)^{2}
$$

and in fact $I(n)=\pi / 2$ if $n=n^{\prime}:=1 /\left(1-\kappa(\pi / 2)^{2}\right)>1$.

In summary the equilibrium equations in this example have the equilibrium pairs $(n, \rho) \in B$ given by $\rho(a)=(n-1)(1-a)(n \kappa)^{-1} \cos I(n) a, 1<n$, where $I(n)$ satisfies (4.1) with $I_{0}>\pi / 2$ when $\kappa$ satisfies (4.2). This set of equilibrium pairs is $C^{+}$in Theorem 1. But note that while $(n, \rho)$ is positive for $1<n<n^{\prime}, \rho(a)$ assumes negative values on $[0, A)$ for $n>n^{\prime}$.

Although the global positivity of the unbounded continuum $\mathrm{C}^{+}$cannot in general be guaranteed there is an important special case when it can, namely the case when the growth rate $\delta$ in (1.2) has the form $\delta=\rho D(\rho)$. The reason for this is that in this case all nontrivial solutions of $(1.3 \mathrm{a})$ are then necessarily of one sign. 
Theorem 2 (Positivity). Assume H1. (i) The equilibrium pairs from $\mathrm{C}^{+}$lying sufficiently close to $(1,0)$ are positive.

(ii) If $h_{2}=\rho d(n, \rho)$ where $d: P \rightarrow C_{0}(A)$ in $H 1$, then all nontrivial equilibrium pairs from $C^{+}$are positive, i.e. $C^{+}=C_{+}^{+} \cup\{(1,0)\}, C_{+}^{+} \subset P^{+}$.

Proof. We need only prove (ii). Let $C_{+}^{+} \subset P^{+}$denote the subset of all positive equilibrium pairs on $C^{+}$and let $C_{-}^{+}$denote the subset of all negative pairs. Any solution of (1.3a) must satisfy

$$
\rho(a)=\rho(0) \rho_{0}(a) \exp \left(-\int_{0}^{a} d(\rho, n) / v d s\right), \quad 0 \leqslant a \leqslant A
$$

and consequently any nontrivial solution has one sign on $[0, A)$. It follows that the only pairs lying in $\mathrm{cl}\left(C_{+}^{+}\right)$or in $\mathrm{cl}\left(C_{-}^{+}\right)$which do not also lie in $C_{+}^{+}$or in $C_{-}^{+}$ respectively are trivial. But the only trivial pair in $C^{+}$is $(1,0)$ and by Theorem 1 it lies in $c l\left(C_{+}^{+}\right)$and not in $\operatorname{cl}\left(C_{-}^{+}\right)$. It follows that $\operatorname{cl}\left(C_{+}^{+}\right)=C_{+}^{+} \cup\{(1,0)\}$, $c l\left(C_{-}^{+}\right)=C_{-}^{+}$and hence $c l\left(C_{+}^{+}\right) \cap \operatorname{cl}\left(C_{-}^{+}\right)=\varnothing$. But clearly $C^{+}$being closed implies $\operatorname{cl}\left(C_{+}^{+}\right) \cup \operatorname{cl}\left(C_{-}^{+}\right) \subseteq C^{+}$. On the other hand if $(n, \rho) \in C^{+}$then either $\rho \equiv 0$ or $\rho$ has one sign so that either $(n, \rho)=(1,0)$ or $(n, \rho)$ lies in $C_{+}^{+} \cup C_{-}^{+}$, i.e. $(n, \rho) \in \operatorname{cl}\left(C_{+}^{+}\right) \cup$

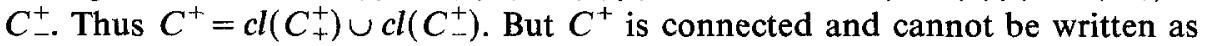
the union of disjoint, nonempty closed sets. Since $c l\left(C_{+}^{+}\right)$contains $(1,0)$, it follows that $c l\left(C_{-}^{+}\right)=\varnothing$ and $C^{+}=\operatorname{cl}\left(C_{+}^{+}\right)=C_{+}^{+} \cup\{(1,0)\}$.

\section{Stability}

In this section the stability of the equilibrium pairs near the bifurcation point $(1,0)$ will be investigated by means of the familiar principle of linearized stability. Solutions of the equations linearized at $(n, \rho)$ of the form $y(a) \exp (z t), 0 \neq y \in B$ and $z=$ complex, will be sought. If such a solution exists for a $z$ with $\operatorname{Re} z>0$ then $\rho$ will be called unstable while if no such solution exists with $\operatorname{Re} z \geqslant 0$ then $\rho$ will be called stable. See $[32,46]$ for a general treatment of this method for some equations of type (1.2).

If Eqs. (1.2) with $\delta=\mu \rho+h_{2}(n, \rho), \beta=n m(\rho)+h_{1}(n, \rho)$ are linearized at a positive equilibrium pair $(n(\varepsilon), \rho(\varepsilon)) \in C^{+}$from Theorem 1 and $\rho=y(a) \exp (z t)$ is substituted into the result, the linear equations

$$
\begin{gathered}
\partial_{a}(v y)+(\mu+z) y+h_{2}^{\prime}(\varepsilon)(y)=0 \\
y(0)=n(\varepsilon) m(y)+h_{1}^{\prime}(\varepsilon)(y)
\end{gathered}
$$

for $0 \neq y \in B$ are obtained where

$$
h_{i}^{\prime}(\varepsilon)(y):=\partial_{\rho} h_{i}(n(\varepsilon), \rho(\varepsilon))(y) .
$$

A linearization at a trivial pair $(n, 0)$ results in the equations

$$
\begin{gathered}
\partial_{a}(v y)+(\mu+z) y=0 \\
y(0)=n m(y) .
\end{gathered}
$$

We are interested in those complex $z$ for which (5.1) and (5.3) have nontrivial solutions $0 \neq y \in B$. 
Consider first Eqs. (5.3) associated with the trivial pair $(n, 0)$. By the theory in Sect. 2, (5.3) has a nontrivial solution if and only if $z$ solves the characteristic equation

$$
n c(z)=1 \quad \text { where } c(z):=m\left(\rho_{0}(a) e^{-z V(a)}\right) .
$$

By $(3.1), c(0)=1$ and if

H3. $m\left(\rho_{0}(a) V(a)\right)>0$.

holds then a simple application of the implicit function theorem implies the existence of a unique (real) solution $z=z(n), z(1)=0$, of (5.4) for $|n-1|$ sufficiently small. An implicit differentiation of $n c(z(n))=1$ shows $\partial_{n} z(0)=$ $1 / m\left(\rho_{0} V\right)>0$ and

$$
z(n)>0 \text { when } n>1, z(n)<0 \text { when } n<1 \text { for } n \sim 1 .
$$

Thus $\rho \equiv 0$ is unstable for $n>1$ and $n \sim 1$. Also $\rho \equiv 0$ is stable for $n<1$ and $n \sim 1$ provided the existence of any other roots satisfying $\operatorname{Re} z \geqslant 0$ (i.e. other than those on the branch $z(n)$ near $z=0$ ) can be ruled out. As we shall see below this will require a further hypothesis on $m$.

The next Lemma shows that $H 3$ similarly guarantees the existence of a locally unique branch of eigenvalues $z=z(\varepsilon)$ of the linearization (5.1)-(5.2) associated with the nontrivial solutions $(n(\varepsilon), \rho(\varepsilon))$ near $z=0$ for $|\varepsilon|$ small. In the age structure case $v \equiv 1$ under (1.4) the number $m\left(\rho_{0} V\right)$ is sometimes referred to as the "mean age of reproduction" [20] and $H 3$ is the reasonable assumption that this mean is positive. The next Lemma also shows how this hypothesis allows us to obtain a relationship between stability and the direction of bifurcation as determined by the constant $\gamma_{1}$ in Theorem 1 .

Lemma. Assume $\mathrm{H} 2$ and H3. There exist positive reals $r>0, \hat{\varepsilon}>0$ such that for $|\varepsilon|<\hat{\varepsilon}$ there exists a unique "eigenvalue" $z$ satisfying $|z|<r$ for which the equations (5.1)-(5.2) have a nontrivial "eigensolution" $y \in B$. This eigenpair has the form $z=z(\varepsilon), y=\rho_{0}+u(\varepsilon)$ where $z:(-\hat{\varepsilon}, \hat{\varepsilon}) \rightarrow R, u:(-\hat{\varepsilon}, \hat{\varepsilon}) \rightarrow B_{0}$ are $q-1$ times continuously differentiable and $z(0)=0, u(0)=0$. Moreover

$$
z_{1}:=\partial_{\varepsilon} z(0)=-\gamma_{1} / m\left(\rho_{0}(a) V(a)\right) .
$$

The proof of this Lemma appears in the Appendix.

Remark. As observed in Remark (4) of Sect. 3 in the case (1.4) only one continuous derivative of $d$ and $r$ are needed near $\rho \equiv 0$ for this Lemma (instead of two or more as implied by $\mathrm{H} 2$ ).

Similar to $(5.5)$ for the trivial equilibria we have from this Lemma that

$$
z(\varepsilon)>0 \quad \text { when } \gamma_{1}<0, \quad z(\varepsilon)<0 \quad \text { when } \gamma_{1}>0 \quad \text { for } \varepsilon \sim 0, \quad \varepsilon>0 .
$$

The positive equilibrium $\rho(\varepsilon)$ is consequently unstable when $\gamma_{1}<0$ and $\varepsilon$ is small. Also $\rho(\varepsilon)$ is stable when $\gamma_{1}>0$ and $\varepsilon$ is small provided once again the existence of eigenvalues $z$ satisfying $\operatorname{Re} z \geqslant 0$ other than those near $z=0$ in the Lemma can be ruled out. For this purpose we assume

H4. $1 \notin c l\{c(z)|\operatorname{Re} z \geqslant 0| z \mid, \geqslant \zeta\}$ for all $\zeta \in R, \zeta>0$. 
Theorem 3 (Stability). Assume H2, H3 and $\gamma_{1} \neq 0$.

(i) For $n>1, n \sim 1$ the trivial equilibrium $\rho \equiv 0$ is unstable. If the positive equilibria in Theorem 1 bifurcate subcritically $\left(\right.$ i.e. $\gamma_{1}<0$ ) then they are unstable for $\varepsilon \sim 0, \varepsilon>0$ (i.e. for $n<1, n \sim 1$ ).

(ii) Assume in addition that $H 4$ holds. Then for $n<1, n \sim 1$ the trivial equilibrium $\rho \equiv 0$ is stable. If the positive equilibria in Theorem 1 bifurcate supercritically (i.e. $\gamma_{1}>0$ ) then they are stable for $\varepsilon \sim 0, \varepsilon>0$ (i.e. for $n>1, n \sim 1$ ).

The proof appears in the Appendix.

Remark. Again only one continuous derivative is needed for $r$ and $d$ in the case (1.4) (instead of two or more as implied by $H 2$ ).

In most applications the linear functional $m$ has an integral form

$$
m(\rho)=\int_{0}^{A} \phi(a) \rho(a) d a, \quad 0 \leqslant \phi \in L(A) .
$$

The condition (3.1) is satisfied in this case if

$$
\int_{0}^{A} \phi(a) \rho_{0}(a) d a=1
$$

For this case

$$
c(z)=\int_{0}^{A} \phi(a) \rho_{0}(a) e^{-z V(a)} d a
$$

satisfies $|c(z)|<1$ for $\operatorname{Re} z>0$ and $c(i \theta) \neq 1, \theta \neq 0$, by (5.8). These facts together with $|c(z)| \rightarrow 0$ as $|z| \rightarrow+\infty, \operatorname{Re} z \geqslant 0$ (which can be shown with the help of the Riemann-Lebesque theorem) show that $\mathrm{H} 4$ always holds for functionals $m$ given by (5.7).

In fact for the case (5.7) the stability and instability assertions for the trivial equilibrium in Theorem 3 when $n$ is near 1 can be shown to hold globally, i.e. $\rho \equiv 0$ is stable for all $n<1$ and unstable for all $n>1$. Indeed, for $n>1$ the expression $n c(z)$ for real $z$ is strictly monotonically decreasing in $z$ from the value $n$ at $z=0$ to 0 at $z=+\infty$ and hence must equal 1 for some positive real $z$, i.e. the characteristic equation (5.4) has a positive real root whenever $n>1$. On the other hand, when $n<1,|n c(z)| \leqslant n<1$ for $\operatorname{Re} z \geqslant 0$ and (5.4) can have no roots with $\operatorname{Re} z \geqslant 0$ for any $n<1$.

Theorem 3 shows that the number $\gamma_{1}$ given by (3.3) is important in determining the stability of the positive bifurcating equilibria, at least near the bifurcation point $(1,0)$. Formula (3.3) relates the stability properties near bifurcation to the second order derivatives of the nonlinearities in the equilibrium equations (1.3). In the important case when the vital rates $\delta$ and $\beta$ have the form (1.4) given by per unit rates $D$ and $F=n f$ the formula (3.3) for $\gamma_{1}$ reduces to

$$
\gamma_{1}=\int_{0}^{A} \phi(a) \rho_{0}(a) \int_{0}^{a} \partial_{\rho} D(0)\left(\rho_{0}\right) / v d s d a-\int_{0}^{A} \rho_{0}(a) \partial_{\rho} f(0)\left(\rho_{0}\right) d a .
$$

Here $m$ has the form (5.7) with $\phi=f(0)$ and $\mu=D(0)$. This is the formula given in [5] when $v \equiv 1$. It shows how the direction of bifurcation and hence stability 
properties of the positive bifurcating equilibria depend on the (lowest order) density effects on the per unit vital rates $D$ and $f$ as measured by the Fréchet derivatives at $\rho \equiv 0$.

\section{The spectrum of $\mathrm{C}_{+}^{+}$}

By Theorem 1 either the spectrum

$$
\sigma=\left\{n \in R \mid(n, \rho) \in C_{+}^{+} \quad \text { for some } \rho \in B\right\}
$$

associated with the positive equilibrium pairs from the continuum $C^{+}$is unbounded or the set of positive equilibria

$$
\Sigma=\left\{\rho \in B \mid(n, \rho) \in C_{+}^{+} \quad \text { for some } n \in \sigma\right\}
$$

is unbounded or both. The closure $\operatorname{cl}(\sigma)$ of $\sigma$ is a closed interval of reals which we denote by $\operatorname{cl}(\sigma)=\left[\sigma_{i}, \sigma_{s}\right]$. Since $(1,0) \in C^{+}, \sigma_{i} \leqslant 1 \leqslant \sigma_{s}$.

In this section some results concerning $\sigma_{i}$ and $\sigma_{s}$ will be obtained for the case (1.4). Specifically we assume

H5. $\delta, \beta$ satisfy $H 1$ and have the form (1.4) with $D(\rho)=\mu+d(\rho)$ where $d: B \rightarrow$ $C_{0}(A)$ is continuous with $d(0)=0$ and $F=n f$ where $f(\rho)=\phi+r(\rho), \phi \in L(A)$, $r: B \rightarrow L(A)$ is continuous with $r(0)=0$.

Theorems 1(i) and 2(ii) imply the existence of an unbounded continuum of equilibrium pairs $C^{+}$. Equation (1.3a) implies for $(n, \rho) \in C_{+}^{+}$that

$$
\rho(a)=\rho(0) \rho_{0}(a) \exp \left(-\int_{0}^{a} d(\rho) / v d s\right), \quad \rho(0)>0
$$

while (1.3b) in turns implies that

where

$$
1=n N(\rho)
$$

$$
N(\rho):=\int_{0}^{A} f(\rho) \rho_{0}(a) \exp \left(-\int_{0}^{a} d(\rho) / v d s\right) d a .
$$

Thus $n N(\rho)$ is an invariant along $C_{+}^{+}$. The identity (6.1), which in fact holds for any nontrivial equilibrium pair, expresses the fact that the birth processes yield exact replacement when the population is held at equilibrium.

The identity (6.1) implies $n \neq 0$ for $n \in \sigma$ and hence $\sigma_{i} \geqslant 0$. In general the endpoints $\sigma_{i}, \sigma_{s}$ can assume any values in the range

$$
0 \leqslant \sigma_{i} \leqslant 1 \leqslant \sigma_{s} \leqslant+\infty .
$$

Properties of $N$ as a functional of $\rho$ (which depend on the properties of the vital rates $D$ and $f$ as operators acting on $\rho$ ) together with (6.1) can be used to obtain information about the spectral interval. Define

$$
N_{i}:=\inf _{\Sigma} N(\rho), \quad N_{s}:=\sup _{\Sigma} N(\rho) .
$$

Since $n>0$ for $n \in \sigma$ and since $N(0)=1$ by (3.1) it follows that

$$
0 \leqslant N_{i} \leqslant 1 \leqslant N_{s} \leqslant+\infty \text {. }
$$


If $\rho^{j} \in \Sigma$ is a sequence for which $N\left(\rho^{j}\right) \rightarrow N_{i}$ then from (6.1) it follows that $n^{j} \rightarrow 1 / N_{i}\left(+\infty\right.$ if $\left.N_{i}=0\right)$ where $n^{j} \in \sigma$ is such that $\left(n^{j}, \rho^{j}\right) \in C_{+}^{+}$. Thus $1 / N_{i} \leqslant \sigma_{s}$. On the other hand if $n^{j} \in \sigma$ is a sequence for which $n^{j} \rightarrow \sigma_{s}$ then $N\left(\rho^{j}\right) \rightarrow 1 / \sigma_{s}(+\infty$ if $\sigma_{s}=0$ ) and hence $1 / \sigma_{s} \geqslant N_{i}$. As a result we conclude that $\sigma_{s}=1 / N_{i}$. Similarly it can be shown that $\sigma_{i}=1 / N_{s}$.

Theorem 4. Assume H5. Then $0 \leqslant \sigma_{i}=1 / N_{s} \leqslant 1 \leqslant \sigma_{s}=1 / N_{i} \leqslant+\infty$.

In most model equations the coefficient $D$ is nonnegative, i.e. $\delta$ is purely a death rate. Also $f$, representing a per unit fertility coefficient, is also usually nonnegative. A common modeling assumption is that the effects of population density cannot increase fertility nor decrease the death rate:

$$
0 \leqslant f(\rho) \leqslant f(0), \quad 0 \leqslant \mu=D(0) \leqslant D(\rho) \quad \text { on }[0, A] \text { for } \rho \in B^{+} .
$$

Then $0 \leqslant N(\rho) \leqslant N(0)=1, \rho \in B^{+}$, which implies $N_{s} \leqslant 1$. Theorem 4 then implies $\sigma_{i}=1$ which means the spectral interval for $C_{+}^{+}$has the form $\sigma=\left(1, \sigma_{s}\right], \sigma_{s} \leqslant+\infty$. In particular, supercritical stable bifurcation always occurs in this case.

It is also often assumed that either the fertility coefficient $f$ drops to 0 or that the death rate $D$ (or equivalently $d$ ) increases without bound with unbounded increases in density. In these cases it can usually be shown that $N(\rho)$ decreases to 0 as density increases without bound; hence $N_{i}=0$ and $\sigma_{s}=+\infty$ by Theorem 4.

Thus for models with the types of density dependent properties described above it is usually the case that the spectral interval for the branch of positive equilibrium pairs $C_{+}^{+}$is $\sigma=(1,+\infty)$, i.e. at least one positive equilibrium exists for each $n>1$ and those pairs $(n, \rho)$ near $(1,0)$ are stable. One cannot necessarily assert however that no other positive equilibria exist, or even that the branch equilibria are unique for a given $n>1$, nor that stability persists globally along the branch.

More detailed information about the spectrum and the question of uniqueness can be obtained from Theorem 4 when the density dependence in the vital rates $\delta, \beta$ is through a dependence on a common linear functional of density. This important special case is considered in the next section.

Remark. Theorem 4 remains valid in the more general case when

$$
\beta=l(\rho f(\rho))
$$

provided $l$ is a bounded linear functional on a suitable Banach space so that $\beta$ satisfies $H 1$. The comments following Theorem 4 also hold if $l$ is monotone. An example is the case when fertility is concentrated in one class of type $a=T$ and $\beta=\left.n \rho f(\rho)\right|_{a=T}$ in which case $l(\rho)=\rho(T)$.

\section{Models utilizing linear functionals of density}

For $n \in \sigma$ there exists at least one positive solution of the equilibrium equations (1.3). The invariance principle (6.1) can often be used to prove uniqueness. For example, if $N(\rho)$ has the property that $N\left(\rho_{1}\right)=N\left(\rho_{2}\right), \rho_{i} \in B^{+}$, implies $\rho_{1}=\rho_{2}$ 
then exactly one positive equilibrium exists in $B^{+}$for each $n \in \sigma$. This is essentially the method of proving uniqueness in many papers in the literature.

Most models of population growth which appear in the literature assume that $D$ and $F=n f$ depend on a linear functional of density $\rho$. Examples of such functionals are total population size $\int_{0}^{A} \rho(a) d a[8,16,17,20,25,26,45]$, more general weighted integrals $\int_{0}^{A} w(a) \rho(a) d a[10,11,14,34,35,45,47]$ and point evaluations $\rho(T)[3,20,29,43]$. In this section we study the unbounded branch. of positive equilibrium pairs from Theorem 1 in more detail using the invariance principle (6.1) for this important special case. Specifically we assume that

H6. Hypothesis $H 5$ holds with $(\mu, v) \in \Delta^{+}(A), 0 \leqslant \phi \in L(A)$ and

(a) $d(\rho)=\hat{d}(p(\rho))$ where $\hat{d}: R \rightarrow C_{0}(A)$ is continuous with $\hat{d}(0)=0$ and $\hat{d}(x) \geqslant 0$ for $x \geqslant 0$

(b) $r(\rho)=\hat{r}(p(\rho))$ where $\hat{r}$ : $R \rightarrow L(A)$ is continuous with $\hat{r}(0)=0$ and $\hat{r}(x) \geqslant 0$ a.e. for $x \geqslant 0$

where $p: B(\mu, v) \rightarrow R$ is linear and bounded and satisfies $p(\rho)>0$ for $\rho \in B^{+}(\mu, v)$.

The normalization (3.1) implies $\int_{0}^{A} \phi(a) \rho_{0}(a) d a=1$ and hence $\phi \neq \equiv$. Thus H6 implies $H 3$.

Set $\hat{D}=\mu+\hat{d}, \hat{f}=\phi+\hat{r}$. Theorems $1(\mathrm{i})$ and 2(ii) can be applied to the equilibrium equations

$$
\begin{aligned}
& \text { (a) } \partial_{a}(v \rho)+\hat{D}(p(\rho)) \rho=0 \\
& \text { (b) } \rho(0)=n \int_{0}^{A} \hat{f}(p(\rho)) \rho(a) d a
\end{aligned}
$$

to obtain an unbounded continuum $C^{+}=C_{+}^{+} \cup\{(1,0)\}$ where $C_{+}^{+}$consists of positive equilibrium pairs. The identity $(6.1)$ for $(n, \rho) \in C_{+}^{+}$becomes

$$
1=n \hat{N}(p(\rho))
$$

where

$$
\hat{N}(x):=\int_{0}^{A} \hat{f}(x) \rho_{0} \exp \left(-\int_{0}^{a} \hat{d}(x) / v d s\right) d a
$$

defines a continuous real valued function $\hat{N}: R \rightarrow R^{+}$which by the normalization (3.1) satisfies $\hat{N}(0)=1$.

In bifurcation problems diagrams depicting the equilibria as functions of $n$ are usually drawn by plotting some measure of the "size" of $\rho$, say $|\rho|_{\mu}$, against $n$. Given the identity (7.2) it is convenient for the equations (7.1) to plot $p(\rho)$ against $n$. The following Lemma shows that the resulting graph accurately displays the uniqueness and boundedness properties of the continuum $C^{+}$. Note that the nonnegativity condition $\hat{D} \geqslant 0$ in $H 5$ implies that $\rho(a) / \rho_{0}(a)$ is noninceasing and hence that $|\rho|_{\mu}=\rho(0)$ for positive equilibria.

Lemma. Assume H6(a). If $\rho_{1}, \rho_{2} \in B$ satisfy (7.1a) and $p\left(\rho_{1}\right)=p\left(\rho_{2}\right)$ then $\rho_{1} \equiv \rho_{2}$.

Assume H6. $\Sigma$ is unbounded if and only if the set $\Pi=\{p(\rho) \mid \rho \in \Sigma\}$ is unbounded. Thus the graph $G=\left\{(n, p(\rho)) \mid(n, \rho) \in C^{+}\right\}$is an unbounded continuum in $R^{+} \times R^{+}$ containing $(1,0)$ for which $(n, p) \in G /\{(1,0)\}$ implies $p>0$. 
Proof. Assume $p\left(\rho_{1}\right)=p\left(\rho_{2}\right)$ and set $e(a)=\exp \left(-\int_{0}^{a} \hat{D} / v d s\right)$ where $\hat{D}=$ $\hat{D}\left(p\left(\rho_{1}\right)\right)=\hat{D}\left(p\left(\rho_{2}\right)\right)$. Then by (7.1a), $\rho_{i}(a)=\rho_{i}(0) e(a)$ and hence $\rho_{1}(0) p(e)=$ $\rho_{2}(0) p(e)$. Since $p(e)>0$ by $H 6$ it follows that $\rho_{1}(0)=\rho_{2}(0)$ and hence $\rho_{1}(a) \equiv \rho_{2}(a)$.

If $\Pi$ is unbounded then the boundedness of $p$ implies $\Sigma$ is unbounded. Conversely suppose $\Sigma$ is unbounded. For purposes of contradiction assume $\Pi$ is bounded. Pick a sequence $\rho^{j} \in \Sigma$ for which $\left|\rho^{j}\right|_{\mu}=\rho^{j}(0) \rightarrow+\infty$ and let $p^{\prime}=$ lim sup $p\left(\rho^{j}\right)>0$. Since $\Pi$ is bounded, $p^{\prime}<+\infty$ and by extracting a subsequence if necessary $p\left(\rho^{j}\right) \rightarrow p^{\prime}$. Equation (7.1a) implies $\rho^{j}(a)=\rho^{j}(0) \rho_{0}(a) e^{j}(a)$ where $e^{j}=\exp \left(-\int_{0}^{a} \hat{d}\left(p\left(\rho^{j}\right)\right) / v d s\right)$. Now $\rho_{0}(a) e^{j}(a) \rightarrow \rho_{0}(a) e^{\prime}(a)$ in $B$ where $e^{\prime}=$ $\exp \left(-\int_{0}^{a} \hat{d}\left(p^{\prime}\right) / v d s\right)$ and consequently we get $p\left(\rho_{0} e^{\prime}\right)=\lim p\left(\rho_{0} e^{j}\right)=$ $\lim p\left(\rho^{j}\right) / \rho^{j}(0)=0$, which contradicts $p\left(\rho_{0} e^{\prime}\right)>0$ from $H 6$.

By (7.2) the graph $G$ must lie on the graph $\hat{G}=\left\{(n, x) \in R^{+} \times R^{+} \mid n \hat{N}(x)=1\right\}$. By plotting this planar graph $\hat{G}$ associated with the real valued function $\hat{N}(x)$ one can get detailed results concerning the spectrum and the uniqueness of positive equilibria. The following theorem contains some simple, but useful results obtained in this way.

Theorem 5. Assume H6 and let $C^{+}=C_{+}^{+} \cup\{(1,0)\}$ be the unbounded continuum of equilibrium pairs from Theorems $1(\mathrm{i})$ and 2(ii). Let $\sigma_{i}, \sigma_{s}$ be the endpoints of the closure of the spectral interval associated with $C_{+}^{+}$.

(i) If $\hat{N}(x)$ is strictly monotone then the equilibrium equations (7.1) have at most one positive equilibrium for any $n \in R$.

(ii) If $\hat{N}(x)<1$ for $x \geqslant 0$ then $C^{+}$bifurcates supercritically. If $\hat{N}(x) \leqslant 1$ for all $x \geqslant 0$ then $\sigma_{i}=1$.

(iii) If $\hat{N}(x)>1$ for $x \geqslant 0$ then $C^{+}$bifurcates subcritically and $0 \leqslant \sigma_{i}<1$. If $\hat{N}(x)$ is bounded for $x \geqslant 0$ then $\sigma_{i}>0$.

(iv) $\hat{N}(+\infty)=0$ implies $\sigma_{s}=+\infty$.

(v) If there exist two constants $0<x_{1}<x_{2} \leqslant+\infty$ such that $\hat{N}(x)$ is strictly increasing for $0<x<x_{1}$ and strictly decreasing for $x_{1}<x<x_{2}$ with $\hat{N}\left(x_{2}-\right)=0$ then (7.1) has exactly two positive equilibria for $\sigma_{i}<n<1$ where $\sigma_{i}=1 / \hat{N}\left(x_{1}\right)$ and exactly one positive equilibrium for all $n>1$. Moreover $C_{+}^{+}$bifurcates subcritically and $p(\rho) \rightarrow x_{2}$ as $n \rightarrow+\infty,(n, \rho) \in C_{+}^{+}$.

These results follow easily from the preceding Lemma, the identity (7.2) and the unboundedness of $C^{+}$(which means either $\sigma_{s}=+\infty$ or $\Sigma$ is unbounded). We omit the details except to point out that $(v)$, which is a case of nonuniqueness and a possible hysteresis phenomenon, follows from the plot of the graph $\hat{G}$ as shown in Fig. 1. In this case the "lower" subcritically bifurcating branch is unstable, at least near $n=1$. It is frequently the case in such cases when the bifurcating branch "turns back" that the "upper" branch consists of stable positive equilibria, although we have no such stability results here. When this occurs then there exist stable positive equilibria for values of the inherent birth modulus $n$ less than 1 (representing per unit replacement at low densities) but the population is subject to a sudden collapse as $n$ is decreased below the critical value $\sigma_{i}$. This hysteresis phenomenon plays an important role in the dynamics of fisheries in the form of so-called "depensation" [1]. We give an example in the next section. 
Fig. 1. The graph $\hat{G}$ is shown under the assumptions of Theorem 5(v)

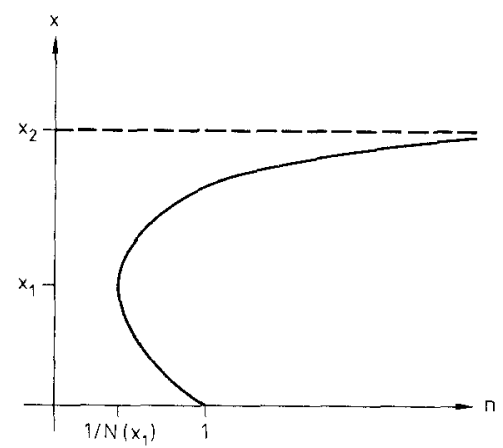

In part (v) the graph of $\hat{N}(x)$ has one "hump". It is graphically clear that the same methods show that when $\hat{N}$ has more humps and depressions then the bifurcating branch turns back several times with the result that possibly more than two positive equilibria exist for certain values of $n \in \sigma$. One expects a change of stability properties with each turn.

Note that the commonly occurring case (6.3) implies by part (ii) that $\sigma_{i}=1$. For this case superctitical bifurcation always occurs and in particular the type of hysteresis described in (v) cannot occur.

As in Sect. 6 the results in Theorem 5 can straightforwardly be extended to the more general case (6.4).

\section{Some examples and applications}

In this section some applications of Theorems 1-5 will be made to model equations which have appeared in the literature and to some generalizations. In these examples $v \equiv 1$ and $\delta, \beta$ have the form (1.4) with $F=n f$. In all cases Theorems 1 and 3 will imply the existence of an unbounded continuum $C^{+}=C_{+}^{+} \cup\{(1,0)\}$ in which $C_{+}^{+}$consists of positive equilibrium pairs $(n, \rho) \in P^{+}$. In all but a single exception Theorem 2 will yield local stability or instability near the bifurcation point $(1,0)$. In some examples Theorem 4 will be used to obtain the direction of bifurcation and the unboundedness of the spectrum. Examples will be given which illustrate all cases of Theorem 5 , as well as the use of the graph $\hat{G}$ in cases not covered by Theorem 5 .

\section{Death rates involving functionals of density}

(1) Model equations (7.1) with

$$
\hat{D}=\mu(a)+\hat{d}(p), \quad \hat{f}=\phi(a), \quad p=\int_{0}^{A} \rho d a
$$

in which the density dependent contribution $\hat{d}$ to the per unit death rate $\hat{D}$ is independent of $a$ and in which per unit fertility $\hat{f}$ is not density dependent are sometimes referred to as "logistic type" models and have been studied by several authors $[20,24,25,46]$. This assumption on $\hat{D}$ is thought to be appropriate in a so-called "harsh environment" in which an increase in the death rate due to 
density effects has an equal effect on all age classes [20]. Under the assumptions

$$
\begin{aligned}
& \text { (a) }(\mu, 1) \in \Delta^{+}(A), \quad 0 \leqslant \phi \in L(A), \quad \int_{0}^{A} \phi \rho_{0} d a=1, \quad \rho_{0}=\exp \left(-\int_{0}^{a} \mu d s\right) \\
& \text { (b) } \hat{d}(p)=\gamma(p) \text { with } \gamma: R \rightarrow R, \quad \gamma(x) \geqslant 0 \text { for } x \geqslant 0, \quad \gamma(0)=0
\end{aligned}
$$

Theorem 5 applies with

$$
\hat{N}(x)=\int_{0}^{A} \phi(a) \rho_{0}(a) \exp (-\gamma(x) a) d a .
$$

Since $N(x) \leqslant 1$ for $x \geqslant 0$, Theorem 5(ii) implies $\sigma_{i}=1$ and hence that supercritical bifurcation occurs. If in addition $\partial_{x} \gamma(x)$ is continuous near $x=0$ then the bifurcation is stable and an exchange of stability occurs at $n=1$. In particular, hysteresis of the type described in Theorem 5(v) cannot occur.

The conditions (8.2) are minimal for models of this type. If further assumptions are made concerning the dependence of the death rate on total population size then Theorem 5 can be used to obtain further results concerning the spectrum. For example if

$$
\gamma(+\infty)=+\infty
$$

then $\sigma_{i}=+\infty$ by Theorem 5(iv) and consequently there exists at least one positive equilibrium $\rho$ for each $n>1$ with $p(\rho) \rightarrow+\infty$ as $n \rightarrow+\infty$. By Theorem 5(i) the assumption that $\gamma(x)$ is strictly increasing for $x \geqslant 0$ implies that at most one positive equilibrium exists for any $n$.

When all these requirements are met then there exists exactly one positive equilibrium for each $n>1$ which is stable at least for $n \sim 1$. An example is the linear case $\gamma(x)=\gamma_{0} x, \gamma_{0}>0$ studied by Marcati [25] (who showed global stability in this case).

The results in Theorem 5 are selected results obtainable from an investigation of the graph $\hat{G}$. Other results can often be obtain using this graph. For example suppose $\gamma: R \rightarrow R$ in (8.2b) is replaced by

$$
\gamma:\left(-p_{0}, p_{0}\right) \rightarrow R, \quad p_{0}>0
$$

and (8.3) is replaced by $\gamma\left(p_{0}-\right)=+\infty$. Then $N\left(p_{0}-\right)=0$ and the set $\Pi \subset\left[0, p_{0}\right)$ in the Lemma of Sect. 7. This implies $\sigma$ is unbounded, i.e. $\sigma_{s}=+\infty$. Thus in this case there exists at least one positive equilibrium $\rho$ for each $n>1$ and $p(\rho) \rightarrow p_{0}$ as $n \rightarrow+\infty$.

(2) Because the results in Sect. 7 apply for any positive functional $p(\rho)$, all of the assertions in part (1) above for models involving total population size remain valid for models involving any such functional of density. For example this is true for functionals of the integral form

$$
p(\rho)=\int_{0}^{A} w(a) \rho(a) d a, \quad 0 \leqslant w \in L(A), \quad w \neq 0 .
$$

The weighting function $w$ is often of a specialized form, such as a gamma distribution. For examples of this case see $[3,8,17,19,20,22,38]$. 
The results in part (1) also remain valid if

$$
p(\rho)=\rho(T) \text { for a fixed } T \in[0, A)
$$

i.e. when the density effects on the death rate are due to the density of a single age class $T$ alone.

(3) By means of the results in Sect. 7 and Theorem 5 it is not difficult to extend the results in (1)-(2) above to models in which the density term $d$ also depends on $a$. For example suppose $(8.2 b)$ is replaced by

$$
\begin{aligned}
d(p)=\gamma(a, p), & \gamma:[0, A] \times R \rightarrow R \quad \text { is continuous } \\
\gamma(a, x) \geqslant 0 & \text { for } x \geqslant 0, \quad \gamma(a, 0) \equiv 0 .
\end{aligned}
$$

Then

$$
\hat{N}(x)=\int_{0}^{A} \phi(a) \rho_{0}(a) \exp \left(-\int_{0}^{a} \gamma(s, x) d s\right) d a
$$

and again $\hat{N}(x) \leqslant 1$ so that supercritical bifurcation occurs. If $\partial_{x} \gamma(a, x)$ is continuous near $x=0$ then this bifurcation is stable. Straightforward extensions of the various conditions on $\gamma$ above yield corresponding results for this case. For example, if $\gamma(a, x) \rightarrow+\infty$ as $x \rightarrow+\infty$ uniformly for $a \in[0, A]$ then $\sigma_{s}=+\infty$ and positive equilibria exist for all $n>1$ and $p(\rho) \rightarrow+\infty$ as $n \rightarrow+\infty$.

\section{Fertility rates involving functionals of density}

In the examples considered in I the fertility rate $f$ was independent of density. That fertility can be significantly effected by population density is widely documented in the biological literature and many mathematical models have been proposed and studied with density dependent fertility rates. Suppose that in place of (8.1) we consider the more general case of model equilibrium equations (7.1) with

$$
\hat{D}=\mu(a)+\hat{d}(p), \quad \hat{f}=\hat{f}(p)
$$

where

$$
\begin{array}{lll}
(\mu, 1) \in \Delta^{+}(A), \quad \hat{d}=\gamma(a, p), & \hat{f}=\psi(a, p) & \\
\gamma:[0, A] \times R \rightarrow R \quad \text { is continuous, } & \gamma(a, x) \geqslant 0 & \text { for } x \geqslant 0, \\
\psi(a, \cdot): R \rightarrow L(A) \quad \text { is continuous, } \quad \psi(a, x) \geqslant 0 & \text { for } x \geqslant 0, \\
\phi(a):=\psi(a, 0) \geqslant 0, \quad \int_{0}^{A} \phi \rho_{0} d a=1 . &
\end{array}
$$

Models of this type with functionals of types (8.4) and (8.5) have been studied by many authors $[3,8,10,11,14,16,26,29,34,35,43,45]$.

Theorem 5 applies to this general class of models with an arbitrary positive linear functional $p$ of density with

$$
\hat{N}(x)=\int_{0}^{A} \psi(a, x) \rho_{0}(a) \exp \left(-\int_{0}^{a} \gamma(s, x) d s\right) d a .
$$


It is rather easy to list simple properties of the vital rates $D$ and $f$ (i.e. of $\gamma$ and $\psi$ as functions of $x$ ) which imply the various properties on $\hat{N}$ required for the conclusions in Theorem 5. For example if density effects cannot cause an increase in fertility so that $\psi(a, x) \leqslant \psi(a, 0)=\phi(a)$ for $x \geqslant 0$, then $\hat{N}(x) \leqslant 1$ and supercritical bifurcation occurs. Rather than make such a list, however, we shall consider some specific models in order to illustrate the results and techniques of Sect. 7.

Some models with density dependent fertility which have appeared in the literature are given by $F=\hat{F}(p)$ with $\hat{F}(x)$ given by

Hoppenstaedt: $\hat{F}=k(a)[1-x / K]_{+}, \quad K>0,[x]_{+} \geqslant 0$ for $x \geqslant 0$,

$$
[x]_{+}=0 \text { for } x<0
$$

Chapman: $\quad \hat{F}=b / c$ for $x=0$ and $b(1-\exp (-x / c)) / x$ for $x \neq 0$

Beverton-Holt: $\quad \hat{F}=b /\left(c+x^{\theta}\right), \quad 0<\theta \leqslant 1$

Ricker: $\quad \hat{F}=(b / c) \exp (1-x / c)$

Depensation: $\quad F=b(e+x) /\left(c+x^{2}\right)$

where all constants $b, c$ and $e$ are positive. We rewrite these birth or recruitment laws in the form $\hat{F}=n \hat{f}$ where $\hat{f}=\psi(a, p)$ has the proper normalization (3.1) which in this case is $\int_{0}^{A} \psi(0) \rho_{0} d a=1$. This gives respectively the model equations (7.1) with

$$
\begin{aligned}
& \psi=\phi(a)[1-x / K]_{+}, \quad \phi=k(a) / \int_{0}^{A} k d a, \quad n:=\int_{0}^{A} k d a \\
& \psi=1 / p_{0} \text { for } x=0 \quad \text { and } \quad\left(c / p_{0}\right)(1-\exp (-x / c)) / x \text { for } x \neq 0, \quad n:=b p_{0} / c \\
& \psi=\left(c / p_{0}\right) /\left(c+x^{\theta}\right), \quad 0<\theta \leqslant 1, \quad n:=b p_{0} / c \\
& \psi=\left(1 / p_{0}\right) \exp (1-x / c), \quad n:=b p_{0} / c \\
& \psi=\left(c / e p_{0}\right)(e+x) /\left(c+x^{2}\right), \quad n:=b e p_{0} / c
\end{aligned}
$$

where $p_{0}=\int_{0}^{A} \rho_{0} d a$. Assume first for simplicity (as is often done in these models) that the death rate is density independent $\gamma \equiv 0$. Theorem 5 applies with the respective functions

$$
\begin{aligned}
& \hat{N}(x)=[1-x / K]_{+} \\
& \hat{N}(x)=1 \text { for } x=0 \text { and } c(1-\exp (-x / c)) / x \text { for } x \neq 0 \\
& \hat{N}(x)=c /\left(c+x^{\theta}\right) \\
& \hat{N}(x)=\exp (1-x / c) \\
& \hat{N}(x)=(c / e)(e+x) /\left(c+x^{2}\right) .
\end{aligned}
$$

For the first four models, $\hat{N}(x)$ is strictly decreasing from 1 at $x=0$ to 0 as $x \rightarrow+\infty$. Thus for the Hoppensteadi, Chapman, Beverton-Holt and Ricker models $\sigma=(1,+\infty)$ and supercritical bifurcation occurs at $n=1$ with exactly one positive 
equilibrium existing for each $n>1$. Moreover, $\hat{N}(x) \equiv 0$ for $x \geqslant K$ in Hoppenstaedt's model while $N(x)>0$ for $x>0$ with $N(+\infty)=0$ in the remaining three. Thus $p(\rho) \rightarrow K$ as $n \rightarrow+\infty$ in Hoppenstaedt's model while $p(\rho) \rightarrow+\infty$ as $n \rightarrow+\infty$ in the Chapman, Beverton-Holt and Ricker models. The differentiability at $x=0$ required in $H 2$ for the local stability results of Theorem 2 apply except in the Beverton-Holt model with $\theta<1$ (in which case a vertical bifurcation occurs). Thus in all of the first four models (with $\theta=1$ in the Beverton-Holt case) the unique positive equilibrium is stable for at least $n \sim 1$.

For the depensatory model Theorem $5(\mathrm{v})$ applies because $\hat{N}(x)$ strictly increases for $0<x<x_{1}:=-e+\left(e^{2}+c\right)^{1 / 2}$ and then strictly decreases for $x>x_{1}$ with $N(+\infty)=0$. Thus a subcritical unstable bifurcation occurs and two positive equilibria exist for $\sigma_{i}:=1 / N\left(x_{1}\right)<n<1$ and one positive equilibria exists for each $n>1$.

The depensatory model with $e=0$ appears in $[10,13,21]$. Our results do not apply to this case because there is no linear recruitment term $(m \equiv 0)$.

It is not difficult to see that all of the above results remain valid for the first four models with density dependent death rates $(\gamma \neq 0)$ if the death rates are nondecreasing in $p\left(\partial_{x} \gamma(a, x) \geqslant 0\right.$ in (8.7)). This leads to a modification of $\hat{N}$ in (8.8) by a decreasing factor less than one which has the same properties as the case $\gamma \equiv 0$. For example, in Hoppenstaedt's model $\hat{N}=$ $[1-x / K]_{+} \int_{0}^{A} \phi \rho_{0} \exp \left(-\int_{0}^{a} \gamma(s, x) d s\right) d a$. For the depensatory model however things are more complicated and a death rate density term might destroy the properties of $\hat{N}$ in Theorem $5(\mathrm{v})$ and even cause supercritical bifurcation to occur.

\section{Some models involving two functionals of density}

In some recent studies of cannibalism in which older individuals eat the very young members of the population (such as larvae or eggs) some models have been proposed and studied in which death rates depend on two different functionals of density. In the model of Diekmann et al. [12] fertility is density independent while the death rate depends on two weighted integrals of density, one of which $p_{1}$ measures the effective number of cannibals and the other of which $p_{2}$ measures the effective number of victims. In this model the per capita rates $D$ and $F=n f$ have the form

$$
\begin{aligned}
& f=\phi(a) \geqslant 0, \quad \phi \in L(A), \quad \int_{0}^{A} \phi \rho_{0} d a=1 \\
& \mathrm{D}=\mu(a)+c(a) p_{1}(\rho) \gamma\left(p_{2}(\rho)\right), \quad(\mu, 1) \in \Delta^{+}(A), \quad 0 \leqslant c \in C_{0}(A) \\
& \gamma: R \rightarrow R \quad \text { is continuously differentiable, } \quad \gamma(0)=1, \quad \gamma(x) \geqslant 0 \quad \text { for } x \geqslant 0
\end{aligned}
$$

where we take $p_{i}: B \rightarrow R$ as arbitrary linear, bounded functionals which satisfy $p_{i}(\rho)>0$ for $\rho \in B^{+}$(in [12] $p_{i}$ have the integral form (8.4)).

Since (6.3) holds we conclude, with the help of Theorems 1-3, that a supercritical stable bifurcation of positive equilibria occurs at $n=1$. The time dependent oscillations found in [12] indicates that stability in general is not preserved globally along the unbounded branch of equilibria. 
Our results in Sect. 3-6 Also permit the same conclusions when the fertility rate is density dependent, provided (6.3) holds.

Other models of a similar sort were studied in $[19,20,22,38]$ in which per capita death and fertility rates depend on the two functionals $p_{1}(\rho)=\rho(0)$, $p_{2}(\rho)=\int_{0}^{A} \rho d a$ and our general results in Sect. 3-6 again permit similar conclusions for such models.

\section{Models with cohort density dependence}

Not all density dependent models of age structured population dynamics assume death and fertility rates depend on linear functionals of density. For example, models have been studied in which these vital rates for an age class $a$ depend on the density of age class $a$ alone $[2,39,40]$. Our results in Sect. 3-6 apply straightforwardly to such models. For example, if $D$ and $f$ are given as in (8.6)-(8.7) with the functional $p(\rho)$ replaced by the density $\rho$ then the results of Sect. 3-6 once again yield an unbounded continuum of positive equilibria pairs which bifurcates supercritically and hence stably.

\section{Some other models}

A very general and flexible modeling assumption is that the per capita vital rates depend on density through integrals of the form $\int_{0}^{A} w(a, s) \rho(s) d s$. Here the weighting kernel $w(a, s) \geqslant 0$ describes how the age class $a$ is effected by the density of age class $s$. If $w$ is multiplicatively separable then one has a model depending on a functional of density as in Sect. 7 and parts (I-III) above. There are models in the literature for which this is not the case however (e.g. see $[3,21]$ ).

As a specific example consider the model of Hoppenstaedt [21] in which fertility $F=n f$ has the form

$$
\begin{gathered}
f=\phi(a)\left[1-\int_{0}^{A} w(a, s) \rho(s) d s\right]_{+}, \quad 0 \leqslant \phi \in L(A), \quad \int_{0}^{A} \phi \rho_{0} d a=1 \\
w:[0, A] \times[0, A] \rightarrow R^{+} \text {is continuous, } \quad w \neq 0 .
\end{gathered}
$$

Let $D=\mu+d(\rho),(\mu, 1) \in \Delta^{+}(A)$ satisfy $H 5$ and (6.3). The unbounded continuum of positive equilibrium pairs bifurcates supercritically and hence stably. Moreover for $\rho \in \Sigma, \rho(a)=\rho(0) \rho_{0}(a) \exp \left(-\int_{0}^{a} d(\rho) d s\right)$ and hence $|\rho|_{\mu}=\rho(0)>0$ and

$$
\begin{aligned}
0<N(\rho)= & \int_{0}^{A} \phi(a)\left[1-|\rho|_{\mu} \int_{0}^{A} w \rho_{0} \exp \left(-\int_{0}^{s} d(\rho) d s\right)\right]_{+} \rho_{0} \\
& \cdot \exp \left(-\int_{0}^{a} d(\rho) d s\right) d a<1 .
\end{aligned}
$$

If $\Sigma$ is bounded then $\sigma$ must be unbounded. On the other hand if $\Sigma$ is unbounded it is clear that $N_{i}=0$ and by Theorem 4 that $\sigma_{s}=+\infty$. Thus in either case $\sigma=(1,+\infty)$. As a result at least one positive equilibrium exists for every $n>1$.

Models have also been studied in which fertility is restricted to a single age class $a=T$ (e.g. see [3]). Formally such models substitute a Dirac function $\delta_{T}(a)$ 
at $a=T$ for $\phi(a)$ or in other words have the form (6.4) where the linear functional $l$ is given by $l(\rho)=\rho(T)$. If this is done in Hoppenstaedt's model (8.9) for example Theorems 1-4 still apply and yield the same conclusions as above. One can also formally substitute Dirac functions for the weighting kernel $w$ and arrive at the same results. For example in [3] weight kernels $\delta_{T}(a)$ and $\delta_{a}(s)$ were used.

\section{Concluding remarks}

A common scenario for models of population growth when the dynamics are studied as a function of a selected parameter (usually an inherent net growth modulus of some kind) is that for parameter values less than a critical value the population is subject to extinction while for values greater than the critical value stable positive equilibrium states exist. Stability may not persist however under further increases of the parameter and Hopf-type bifurcations to time periodic limit cycles may occur. It is possible that further increases can lead to repeated "cascading" bifurcations and even to "chaos" in the now familiar manner exhibited by well studied difference equations. Virtually all model equations of population gorwth which appear in the literature can be derived from the fundamental equations (1.2) by making suitable simplifying assumptions on $\delta, \beta$ and appropriate mathematical manipulations ... be they ordinary or functional or delay or integro- differential equations, integral equations or even (by discretation) difference and Lesli matrix-type equations. Given these facts, it is natural to ask to what extent the above described bifurcation scenario is valid for the general system (1.2).

In this paper we have shown that under very general conditions the primary bifurcation of positive equilibria in this scenario always occurs in the general model (1.2), i.e. a global continuum of equilibria bifurcates from the zero density at the unique (normalized) critical value $n=1$ of the net birth modulus $n$. This continuum consists of a branch of positive equilibria near bifurcation and in the important special case of vital rates (1.4) expressed in terms of per unit rates the continuum remains globally positive. It was shown that the zero density loses stability as $n$ increases through $n=1$ and that under suitable local differentiability conditions near the bifurcation point the positive branch equilibria are stable if and only if the bifurcation is supercritical, at least near bifurcation. (Stability may not persist globally; Hopf-type bifurcation to time periodic solutions of the general equation (1.2) was studied in [4].)

The spectrum of $n$ values corresponding to positive branch equilibria was also studied and characterized in a certain way in terms of the density dependence properties of the per capita vital rates. The important special case of models depending on functionals of densities was studied in more detail and it was shown how a bifurcation diagram can be easily drawn using a certain real valued function derived from an invariant which holds on the continuum. These results have widespread applicability to models of population growth as demonstrated by the examples given in Sect. 8 .

The stability and global existence results obtained in this paper can be extended to systems of equations of the form (1.2) to include models of interacting 
populations. This will be the subject of a future paper. Some results for nonautonomous, but time periodic equations and the bifurcation of positive periodic solutions have been obtained in [7].

\section{Appendix}

Proof of Theorem 1. (i) Writing the equilibrium equations (1.3) as (recall $v(0)=1$ )

$$
\begin{aligned}
& \partial_{a}(v \rho)+\mu \rho=g_{2}(\lambda, \rho) \\
& \rho(0)=\frac{1}{2} m(\rho)+\lambda m^{\prime}(\rho)+g_{1}(\lambda, \rho)
\end{aligned}
$$

where $\lambda=n-\frac{1}{2}, g_{2}:=-h_{2}\left(\lambda+\frac{1}{2}, \rho\right)$ and $g_{1}:=h_{1}\left(\lambda+\frac{1}{2}, \rho\right)$ and using the solution operator $S$ from the Lemma of Sect. 2 with $n=\frac{1}{2}$ we see that $(n, \rho) \in P$ is an equilibrium pair if and only if $(\lambda, \rho) \in P$ solves the operator equation

$$
\rho=\lambda L \rho+G(\lambda, \rho)
$$

where $L \rho:=S m^{\prime}(\rho), m^{\prime}(\rho):=(m(\rho), 0) \in P$ and $G:=S g, g:=\left(g_{1}(\lambda, \rho), g_{2}(\lambda, \rho)\right)$. By $H 1, L: B \rightarrow B$ is linear and compact, $G: P \rightarrow B$ is continuous and compact and $|G|_{\mu}=0\left(|\rho|_{\mu}\right)$ near $\rho=0$ uniformly on bounded $n$ intervals. As pointed out in Sect. 3 the only possible trivial equilibrium pair $(n, 0)$ which can lie in $\operatorname{cl}(E)$ is $(1,0)$. As a result either $\operatorname{cl}(E)=E$ or $E \cup\{(1,0)\}$. That the latter holds as well as the rest of the assertions in (i) will follow from the results for equations of the form (A.1) in [33] (specifically Lemma 1.24 and Corollary 1.42) once we have shown that $\lambda=\frac{1}{2}$ is the only characteristic value of $L$ and that it is simple.

The equation $\rho=\lambda L \rho, 0 \neq \rho \in B$, is equivalent to the linear homogeneous system (2.4) and consequently the only characteristic value is $\lambda=\frac{1}{2}$ to which there is associated exactly one independent eigensolution $\rho=\rho_{0}$. To show that $\lambda=\frac{1}{2}$ is simple we must show that any solution of $\left(I-\frac{1}{2} L\right)^{2} \rho=0$ is a solution of $\left(I-\frac{1}{2} L\right) \rho=0$. Suppose $\rho \in B$ satisfies the former equation. Then $\rho^{\prime}=\left(I-\frac{1}{2} L\right) \rho$ satisfies the latter equation and hence $\rho^{\prime}=k \rho_{0}=(k / 2) L \rho_{0}$ for some $k \in R$. This means $\rho=\frac{1}{2} L\left(\rho+k \rho_{0}\right)$ which by the definition of $L$ is equivalent to $\rho \in B$ solving the nonhomogeneous equations (2.2) with $n=1$ and $g=\left(g_{1}, g_{2}\right)=(k / 2,0)$. By the Lemma of Sect. 2 it follows that $0=\Omega(g)=k / 2$. Thus $k=0$ which implies that $0=\left(I-\frac{1}{2} L\right) \rho$ and that $\lambda=\frac{1}{2}$ is simple.

(ii) A substitution of (3.2) into the equilibrium equations (1.3) yields, after a division by $\varepsilon$, the equations

$$
\begin{gathered}
\partial_{a}(v w)+\mu w=H_{2}(\varepsilon, w, \gamma) \\
\left.w\right|_{a=0}=m(w)+H_{1}(\varepsilon, w, \gamma)
\end{gathered}
$$

for $w \in B_{0}, \gamma \in R$ where $H_{1}:\left(-\varepsilon_{0}, \varepsilon_{0}\right) \times B_{0} \times R \rightarrow R, H_{2}:\left(-\varepsilon_{0}, \varepsilon_{0}\right) \times B_{0} \times R \rightarrow B$, as given by $H_{i}=$ $(2-i) \gamma m\left(\rho_{0}+w\right)+(-1)^{i-1} h_{i}\left(1+\gamma, \varepsilon \rho_{0}+\varepsilon w\right) / \varepsilon$, are $q-1 \geqslant 1$ times continuously differentiable near $(\varepsilon, w, \gamma)=(0,0,0)$ and $H_{i}(0, w, \gamma) \equiv 0$. (A.2) can be solved by constructing an equivalent operator equation as follows. Given $w \in B_{0}$ define $\gamma=\gamma(\varepsilon, w) \in R$ so that the necessary solvability condition $\Omega(H)=0$ holds, $H:=\left(H_{1}, H_{2}\right)$; that is the equation

$$
\Gamma_{1}(\varepsilon, w, \gamma):=H_{1}(\varepsilon, w, \gamma)+m\left(\rho_{0}(a) \int_{0}^{a} H_{2}(\varepsilon, w, \gamma) / \rho_{0} v d s\right)=0
$$

is solved for $\gamma$. This can be done for $(\varepsilon, w)$ near $(0,0)$ by means of the implicit function theorem since $\Gamma_{1}(0,0,0)=0, \partial_{\gamma} \Gamma_{1}(0,0,0)=m\left(\rho_{0}\right)=1$. This yields a $q-1$ times continuously differentiable $\gamma=\hat{\gamma}(\varepsilon, w), \hat{\gamma}(0,0)=0$. In fact $\hat{\gamma}(0, w) \equiv 0$.

With $\gamma=\hat{\gamma}(\varepsilon, w)$ in (A.2) these equations can be reformulated as the equivalent equation $\Gamma_{2}(\varepsilon, w):=w-S_{0} H(\varepsilon, w, \hat{\gamma}(\varepsilon, w))=0$ which can be solved by another application of the implicit function theorem since $\Gamma_{2}(0,0)=0, \partial_{w} \Gamma_{2}(0,0)=I$. This results in a $q-1$ times continuously differentiable $w=w(\varepsilon), w(0)=0$ which in turn yields $\gamma=\gamma(\varepsilon)=\hat{\gamma}(\varepsilon, w(\varepsilon))$ for $|\varepsilon|$ small. 
To obtain formula (3.3) divide (A.3) by $\varepsilon$ and let $\varepsilon \rightarrow 0$. (3.3) then follows since by $H 1$

$$
\begin{aligned}
& H_{1} / \varepsilon=\varepsilon^{-1} m\left(\rho_{0}+w\right)+\varepsilon^{-2} h_{1}\left(1+\gamma, \varepsilon \rho_{0}+\varepsilon w\right) \rightarrow \gamma_{1}+\frac{1}{2} \partial_{\rho}^{2} h_{1}(1,0)\left(\rho_{0}, \rho_{0}\right) \\
& H_{2} / \varepsilon=\varepsilon^{-2} h_{2}\left(1+\gamma, \varepsilon \rho_{0}+\varepsilon w\right) \rightarrow \frac{1}{2} \partial_{p}^{2} h_{2}(1,0)\left(\rho_{0}, \rho_{0}\right)
\end{aligned}
$$

Proof of the Lemma in Sect. 5. The existence and uniqueness of the solutions $z, y$ for $|\varepsilon|$ small can be established by a straightforward application of the implicit function theorem (along the lines of the proof above of Theorem 1 (ii)) using the solution operator $S_{0}$ in the Lemma of Sect. 2 with $n=1$ and the expansions (3.2) for $n(\varepsilon)$ and $\rho(\varepsilon)$. The details are omitted.

To derive the formula (5.6) substitute $z=z(\varepsilon), y=\rho_{0}+u(\varepsilon)$ into the linearized equations (5.1) to obtain

$$
\begin{aligned}
\partial_{a}(v u)+\mu u & =-z\left(\rho_{0}+u\right)-h_{2}^{\prime}(\varepsilon)\left(\rho_{0}+u\right) \\
\left.u\right|_{a=0} & =m(u)+\gamma(\varepsilon) m\left(\rho_{0}+u\right)+h_{1}^{\prime}(\varepsilon)\left(\rho_{0}+u\right)
\end{aligned}
$$

for $u \in B_{0}$. From the necessary solvability condition of Sect. 2 follows

$$
z m\left(\rho_{0}\left(V+\int_{0}^{a} u / v d s\right)\right)=\gamma(\varepsilon) m\left(\rho_{0}+u\right)+h_{1}^{\prime}(\varepsilon)\left(\rho_{0}+u\right)-m\left(\rho_{0}(a) \int_{0}^{a} h_{2}^{\prime}(\varepsilon)\left(\rho_{0}+u\right) / \rho_{0} v d s\right)
$$

Dividing this equation by $\varepsilon$ and letting $\varepsilon \rightarrow 0$ one obtains, using the definitions (5.2) of $h_{i}^{\prime}$

$$
z_{1} m\left(\rho_{0} V\right)=\gamma_{1}+\partial_{\rho}^{2} h_{1}(1,0)\left(\rho_{0}, \rho_{0}\right)-m\left(\rho_{0}(a) \int_{0}^{a} \partial_{\rho}^{2} h_{2}(1,0)\left(\rho_{0}, \rho_{0}\right) / \rho_{0} v d s\right)
$$

or in view of the formula (3.3) for $\gamma_{1}$

$$
z_{1} m\left(\rho_{0} V\right)=\gamma_{1}-2 \gamma_{1}=-\gamma_{1}
$$

Proof of Theorem 3. All that needs be proved is (ii) and this will follow from the Lemma of Sect. 5 if it can be shown that for $|\varepsilon|$ small there exist no eigenvalues $z$ satisfying $\operatorname{Re} z \geqslant 0,|z| \geqslant r$.

Consider first the trivial solution $\rho=0$ and its characteristic equation (5.4). For purposes of contradiction suppose there are sequences $n^{j}, z^{j}$ such that $n^{j}<1, n^{j} \rightarrow 1, \operatorname{Re} z^{j} \geqslant 0,\left|z^{j}\right| \geqslant r$ and (5.4) holds for $n=n^{j}, z=z^{j}$. Since $m$ is bounded the set $\{c(z)|\operatorname{Re} z \geqslant 0| z \mid, \geqslant r\}$ is bounded and by extracting a subsequence if necessary we can assume $c\left(z^{j}\right) \rightarrow c^{\prime}$ where $c^{\prime} \neq 1$ by H4. From (5.4) follows the contradiction that $1=n^{j} c\left(z^{j}\right) \rightarrow c^{\prime} \neq 1$.

Similarly for the positive equilibria $\rho(\varepsilon)$ we assume there exist sequences $\varepsilon^{j}, z^{j}$ such that $\varepsilon^{j}>0$, $\varepsilon^{j} \rightarrow 0, \operatorname{Re} z^{j} \geqslant 0,\left|z^{j}\right| \geqslant r$ for which (5.1) has a nontrivial solution $y^{j} \in B$ which without loss in generality satisfies $\left|y^{j}\right|_{\mu}=1$. Again by extracting a subsequence if necessary we assume that $c\left(z^{j}\right) \rightarrow c^{\prime}$ where $c^{\prime} \neq 1$ by $H 4$. Equation (5.1a) for $y=y^{j}$ can be written

$$
y^{j}(a)=\rho^{j}(a) e^{j}(a)\left[y^{j}(0)-\int_{0}^{a} h_{2}^{\prime}\left(\varepsilon^{j}\right)\left(y^{j}\right) / \rho_{0}(s) v(s) e^{j}(s) d s\right]
$$

where $e^{j}(a):=\exp \left(-z^{j} V(a)\right)$ which when substituted into (5.1b) yields

$$
\left(1-n\left(\varepsilon^{j}\right) c\left(z^{j}\right)\right) y^{j}(0)=R^{j}
$$

where

$$
R^{j}=-n\left(\varepsilon^{j}\right) m\left(\rho_{0}(a) e^{j}(a) \int_{0}^{a} h_{2}^{\prime}\left(\varepsilon^{j}\right)\left(y^{j}\right) / \rho_{0}(s) v(s) e^{j}(s) d s\right)+h_{1}^{\prime}\left(\varepsilon^{j}\right)\left(y^{j}\right) \rightarrow 0
$$

as $j \rightarrow+\infty$ by the definition (5.2) of $h_{i}^{\prime}$ and the order properties of $h_{i}$ near $\rho=0$ in H1. (A.5) implies $y^{j}(0) \rightarrow 0$ which by (A.4) in turn implies the contradiction $1=\left|y^{j}\right|_{\mu} \rightarrow 0$.

\section{References}

1. Clark, C. W.: Mathematical Bioeconomics. New York, Wiley 1976

2. Coleman, B. D.: On growth of populations with narrow spread in reproductive age, J. Math. Biol. 6, 1-19 (1978) 
3. Cushing, J. M.: Model stability and maturation periods in age structured populations, J. Theo. Biol. 86, 709-730 (1980)

4. Cushing, J. M.: Bifurcation of time periodic solutions of the McKendrick equations with applications to population dynamics, Comput. Math. Appl. 9, 459 478 (1983)

5. Cushing, J. M.: Existence and stability of equilibria in age-structured population dynamics. J. Math. Biol. 20, 259-276 (1984)

6. Cushing, J. M.: Global branches of equilibrium solutions of the McKendrick equations of age-structured population growth. To appear in Comput. Math. Appl. (1985)

7. Cushing, J. M.: Periodic McKendrick equations for age-structured population growth. To appear in Comput. Math. Appl. (1986)

8. Cushing, J. M.: Volterra Integrodifferential Equations in Population Dynamics. In: (Iannelli, M. ed.) Mathematics in Biology. Editore, Naples: Liguori 1981

9. DiBlasio, G.: Nonlinear age-dependent population growth with history dependent birth rate. Math. Biosci. 46 279-291 (1979)

10. DiBlasio, G.: Asymptotic behavior of an age-structured fish population. Comput. Math. Appl. 9, 377-381 (1983)

11. DiBlasio, G, Iannelli, M., Sinestrari, E.: Approach to equilibrium in age structured populations with an increasing recruitment process. J. Math. Biol. 13, 371-382 (1982)

12. Diekmann, O., Nisbet, R. M., Gurney, W. S. C., van den Bosch, F.: Simple mathematical models for cannibalism: a critique and a new approach. Centre for Math. Comput. Sci., Report no. AM-R8505. Amsterdam 1985

13. Dietz, K.: Transmission and control of arbovirus diseases Proc. SIMS Conf. on Epidemics. Alta Utah 1974

14. Frauenthal, J. C., Swick, K. E.: Stability of biochemical reaction tanks. Comput. Math. Appl., 9, 499-506 (1983)

15. Getz, W.: The ultimate sustainable yield problem in nonlinear age-structured populations. Math. Biosci. 48, 279-292 (1980)

16. Gurtin, M, MacCamy, R. C.: Nonlinear age dependent population dynamics. Arch. Rat. Mech. Anal. 54, 281-300 (1974).

17. Gurtin, M., MacCamy, R. C.: Some simple models for nonlinear age-dependent population dynamics. Math. Biosci. 43, 199-211 (1979)

18. Gurtin, M., Levine, D. S.: On populations which cannibalize their young. SIAM J. Appl. Math. 42, 94-108 (1982)

19. Gurtin, M., Levine, D. S.: On predator-prey interactions with predation dependent on age of prey. Math. Biosci. 47, 207-219 (1979).

20. Gurtin, M. E.: The Mathematical Theory of Age-Structured Populations. Preprint

21. Hoppenstaedt, F.: Mathematical Theories of Populations: Demographics, Genetics and Epidemics. SIAM Conf. Series Appl. Appl. Math., Philadelphia 1975

22. Levine, D. S.: Models of age-dependent predation and cannibalism via the McKendrick equation. Comput. Math. Appl. 9, 403-414 (1983)

23. Levine, D. S.: On the stability of a predator-prey system with egg-eating predators. Math. Biosci. 56, 27-46 (1981)

24. Marcati, P.: Asymptotic behavior in age dependent population dynamics with hereditary renewal law. SIAM J. Math. Anal. 12, 904-916 (1981)

25. Marcati, P.: On the global stability of the logistic age dependent population equation, J. Math. Biol. 15, 215-226 (1982)

26. Marcati, P.: Some considerations on the mathematical approach to nonlinear age dependent population dynamics. Comput. Math. Appl. 9, 361-370 (1983)

27. Murphy, L.: Density dependent cellular growth in an age structured colony. Comput. Math. Appl. 9, 383-392 (1983)

28. Nisbet, R. M., Gurney, W. S. C.: Modelling Fluctuating Populations. New York, Wiley 1982

29. Oster, G.: Lectures in Population Dynamics. Appearing in Lec. Appl. Math. 16, AMS 149-170 (1977)

30. Prüss, J.: Equilibrium solutions of age-specific population dynamics of several species. J. Math. Biol. 11, 65-84 (1981) 
31. Prüss, J.: On the qualitative behaviour of populations with age-specific interactions. Comput. Math. Appl. 9, 327-339 (1983)

32. Prüss, J.: Stability analysis for equilibria in age-specific population dynamics. Nonl. Anal. Th. Math. Appl. 7, 1291-1313 (1983)

33. Rabinowitz, P. H.: Some global results for nonlinear eigenvalue problems. J. Func. Anal. 1, 487-513 (1971)

34. Rorres, C.: Stability of an age specific population with density dependent fertility. Theor. Pop. Biol. 10, 24-46 (1976)

35. Rorres, C.: Local stability of a population with density dependent fertility. Theor. Pop. Biol. 16, 283-300 (1979)

36. Rubinow, S. I.: A maturity-time representation for cell population. Biophys. J. 8, 1055-1073 (1968)

37. Rubinow, S. I.: Age-structured equations in the theory of cell populations. Studies in Math. Biol. II, MAA, Washington, 1978

38. Saleem, M.: Predator-prey relationships: egg-eating predators. J. Math. Biol. 65, 187-197 (1983)

39. Sinestrari, E.: Non-linear age-dependent population growth. J. Math. Biol. 9, 331-345 (1980)

40. Sinestrari, E.: Local and global stability for the solutions of a nonlinear renewal equation. Comput. Math. Appl. 9, 353-360 (1983)

41. Sinko, J. W., Streifer, W.: A new model for the age-size structure of a population. Ecol. 48, 910-918 (1967)

42. Streifer, W.: Realistic models in population ecology. Appearing in: Macfayden, A. (ed.) Advances in Ecology Research, 8. New York: Academic Press 1974

43. Swick, K. E.: A nonlinear age-dependent model of single species population dynamics. SIAM J. Appl. Math. 22, 488-498 (1977)

44. von Foerster, H.: Some remarks on changing populations. Appearing in: Strohlman, Jr., F. (ed.) The Kinetics of Cellular Proliferation, 382-407, New York: Gruen and Stratton 1959

45. Wang, F. J. S.: Stability of an age-dependent population. SIAM J. Math. Anal. 11, 683-689 (1980)

46. Webb, G. F.: Theory of Nonlinear Age-Dependent Population Dynamics. Monographs in Pure and Applied Mathematics Series, 89. New York: Marcel Dekker 1985

47. Webb, G. F.: Logistic models of structured population growth. Preprint

Received May 15, 1985 